\title{
The Violet Flux Deficiency of Cool Carbon Stars
}

\author{
Donald G. Lut termoser Hollis R. Johnson \\ Indiana University
}

A characteristic feature of carbon star spectra is the dramatic decrease in energy flux toward the violet. This flux decreases faster toward shorter wavelengths in $\mathrm{N}$-type carbon stars than in either $\mathrm{M}$ giants or blackbodies of the same usual color temperatures, and an unknown violet opacity source in these carbon stars has consequently been the subject of speculation for the past 60 years. We investigate this longstanding problem of the violet flux falloff in cool carbon stars by directly testing, through synthetic spectra, the effects of many new and previously suggested opacity sources, based on currently available model atmospheres for carbon stars and M-giant stars. While several bound-free edges of neutral metals are important opacities, those of $\mathrm{Na} I$ at $2413 \AA$, Mg I at $2514 \AA$, and $\mathrm{Ca} I$ at $2940 \AA$ are especially significant. Collectively, thousands of atomic lines, and in particular, the neutral metal resonance lines are the primary cause of the violet flux falloff. The severe violet flux decrease begins in cool carbon stars shortward of $4500 \AA$, roughly coincident with the redward wing of the extremely strong $\mathrm{Ca} I$ resonance line $4227 \AA$. The enormous line of $\mathrm{Mg} I$ at $2852 \AA$ is one of the largest opacity sources in the near-ultraviolet region of these stars and influences the spectrum well into the visible. The pseudo-continuum of $\mathrm{C}_{3}$ and the photodissociation continuum of $\mathrm{CH}$ both play noticeable but secondary roles. Other opacities that may affect the violet flux include the photodissociation of other hydrides, particularly $\mathrm{NH}$ and $\mathrm{SiH}$, which have dissociation energies similar to $\mathrm{CH}$. Synthetic spectra from the carbon-star models computed both with and without polyatomic molecules fit nicely to the collected observations of the well-observed carbon star TX Psc.

The severe violet flux fallof of these carbon stars is thus primarily a temperature effect. Carbon abundance plays an indirect role in determining the amount and depth of 00 cooling as can be demonstrated by comparing synthetic spectra of a carbon rich model and an oxygen-rich model with the same effective temperature and surface gravity -- the carbon-rich model is affected much more in the violet from the above mentioned opacity sources. If this scenario is correct, then the severity of the violet flux falloff as function of the "N" spectral type will be sensitive to a combination of both effective temperature and C/O ratio. This is indeed the case (as can be seen by comparing Table 1 and Table 4 of Lambert et al. 1986, Ap. J. Suppl. 62, 373). 